\title{
BRISP, a new small-angle time-of-flight neutron spectrometer to study collective dynamics in disordered matter
}

\author{
A.Orecchini ${ }^{1 *}$,W.-C.Pilgrim ${ }^{2}$, C.Petrillo ${ }^{1}$, J.-B.Suck ${ }^{3}$, F.Sacchetti ${ }^{1}$ \\ ${ }^{1}$ Department of Physics, University of Perugia, I-06123 Perugia, Italy \\ 2 Physical Chemistry, Philipps-University of Marburg, D-35032 Marburg, Germany \\ 3 Institute of Physics, Technical University of Chemnitz, D-09126 Chemnitz, Germany
}

Received November 20, 2007

\begin{abstract}
An outline is given of the new neutron Brillouin Spectrometer BRISP which was installed during the last years and recently became fully operational at the Institute Laue-Langevin in Grenoble (France). The main technical features are described and some of the first experimental results are presented, demonstrating the present capabilities of this new-type inelastic small-angle spectrometer. Future upgrades are foreseen and expected to further improve the instrument performances.
\end{abstract}

Key words: neutron scattering instrumentation, time of flight, small-angle spectrometers

PACS: 07.05.Fb, 29.30. Hs

\section{Introduction}

Inelastic neutron scattering (INS) techniques have played a key role in the experimental determination of particle motion in condensed matter for about 50 years. Different ingenious scattering methods have been developed since then, and modern condensed matter physics greatly profits from the high-quality data that are meanwhile available in modern neutron facilities. About ten years ago another new method was developed to explore the microscopic particle dynamics in condensed matter utilizing highly-brilliant radiation, produced by the so-called third-generation synchrotrons, in combination with sophisticated methods for high-resolution energy discrimination: inelastic x-ray scattering (IXS). Since then IXS has significantly contributed to microscopic dynamics research as well. However, instead of becoming a pure competitive method to INS, IXS rather became a powerful complementary technique and today more and more advantage is taken from the synergetic use of the two techniques. Nevertheless, most inelastic scattering experiments are still carried out with neutrons. This has to do with the unique properties of the neutron allowing to access various microscopic features of condensed matter. For example, while coherently scattered neutrons permit to investigate the relative dynamics of different particles in fluids, solids or molecular subunits in larger molecules, incoherent scattering provides information on the motion of single particles on microscopic space- and timescales. Also, the magnetic moment of the neutron provides the unique possibility to measure the microscopic dynamics of magnetic samples and collective magnetic excitations. Furthermore, with some neutron spectrometers the energy resolution can be driven down to values in the $\mu \mathrm{eV}$ range, or even lower, allowing to determine extremely low-lying excitations and very slow dynamical processes. This is also made possible by the usually very regular shape of the resolution function, whose fast-decaying Gaussian tails ensure proper access to modes close to the elastic peak. This is another feature of neutrons as compared to $\mathrm{x}$-rays, where the resolution is rather Lorentzian-like with wide wings extending into the inelastic part of the spectra.

\footnotetext{
*E-mail: andrea.orecchini@pg.infn.it
} 
However, despite the triumphant procession of neutron scattering which began about half a century ago, there is one serious constraint which has hampered the full access to the microscopic world and which has been an annoying problem to scientists since then: the kinematic restriction of the $Q-E$ plane. This limitation has its origin in the characteristic energy-momentum relation of the neutron in combination with the usually low energies of the incident neutrons. A problem which does not occur in IXS where the incoming energy is about six orders of magnitude larger than the energy transfers of interest. In an inelastic scattering experiment, the relation between the momentum transfer $Q$, the scattering angle $2 \vartheta$ and the energy transfer $E=E_{0}-E_{\mathrm{f}}$, where $E_{\mathrm{f}}$ and $E_{0}$ are the energy of the scattered and the incoming neutron, respectively, is given by

$$
Q^{2}(2 \vartheta, E)=\frac{2 m}{\hbar^{2}}\left[2 E_{0}-E-2 \cos (2 \vartheta) \sqrt{E_{0}\left(E_{0}-E\right)}\right] .
$$

The insertion of the minimum and maximum scattering angles and the value of the incoming neutron energy $E_{0}$ into this expression yields a characteristic kinematic plane for a specific instrument. Scattering data can only be acquired within this plane. However, the smaller the incoming energy of the neutron the larger is the curvature of the plane restricting the accessibility of energy transfers especially at smaller $Q$-values. Figure 1 illustrates this situation. There is shown, as an example,

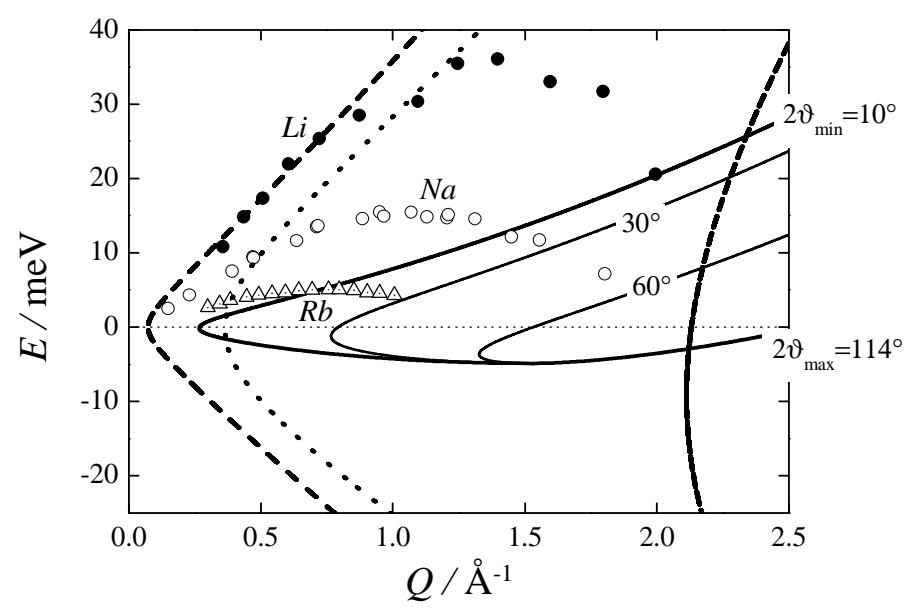

Figure 1. Available $Q-E$ range for different time-of-flight spectrometers (all at the ILL): IN6 (full lines), IN4 (dotted line) and BRISP (dashed lines). Also shown are dispersion relations of the collective excitations in liquid $\mathrm{Li}(\bullet)[1], \mathrm{Na}(\circ)$ [2] and $\mathrm{Rb}(\triangle)$ [3]. The angles reported on the right-hand side of the figure are the minimum and maximum scattering angles available at the IN6 spectrometer.

the kinematic plane of the time-of-flight spectrometer IN6 of the Institute Laue-Langevin (ILL) in Grenoble, one of the most frequently used instruments worldwide, for the largest incoming neutron energy available there $(4.88 \mathrm{meV})$. It is confined by the minimum and maximum scattering angles of $10^{\circ}$ and $114^{\circ}$. The $Q-E$ variation at a constant angle is exemplary shown by two paths at $30^{\circ}$ and $60^{\circ}$, respectively. Also depicted are the $Q-E$ positions of the collective longitudinal excitations for liquid Lithium [1], Sodium [2] and Rubidium [3]. It is apparent that they are hardly accessible with this spectrometer, especially in the lower momentum transfer region, where the energy of the phonon-like modes increases monotonously with rising $Q$. This increase in the lower $Q$-region is approximately determined by the bulk sound velocity in the sample [4]. Since the slope of the instrumental $Q-E$ paths in figure 1 gives the corresponding phase velocity, the low $Q$-part of the dispersion relations can only be measured if the speed of the incoming neutron is larger than the sound velocity in the sample. To improve the kinematic situation, higher incoming neutron energies are needed which, however, shifts the accessible momentum transfer range to higher $Q$. Therefore, it is also mandatory to reduce the minimum scattering angle. The dotted line in figure 1 represents the lower boundary of the kinematic plane for the IN4 spectrometer, also located at the ILL. The 
minimum angle is $2 \vartheta=3^{\circ}$ and the spectrometer can be operated with an incoming energy of $100 \mathrm{meV}$. Nevertheless, the $\mathrm{Na}$ dispersion relation is only partly available and the data for Li are still out of reach.

In order to get access to low- $Q$ inelastic spectra, a new design for a neutron spectrometer has been developed which combines time-of-flight techniques of higher-energy neutrons with smallangle capabilities [5]. This concept has led to the installation of the new Brillouin Spectrometer BRISP which has recently been set up at the high-flux reactor of the Institute Laue-Langevin in Grenoble (France). BRISP is a joint project operated by a collaborative research group (CRG) between the Italian Istituto Nazionale per la Fisica della Materia (INFM), the Technical University of Chemnitz (Germany) and the Philipps University of Marburg (Germany).

With its new capabilities BRISP permits to access a number of scientific problems where spectra over wider energy ranges at low $Q$ are desired: e.g. in the field of microscopic fluid dynamics. Here, a lot of effort has been invested over the past 40 years in order to understand the motion of fluid particles on microscopic time- and lengthscales and to relate this knowledge to the bulk transport properties of the liquid. Especially, collective dynamics has attracted much interest. Characteristic of the correlated collective particle motion are the so-called short-wavelength density-density fluctuations, which lead to distinct excitations in the dynamic scattering law $S(Q, E)$. These excitations may be viewed as the analogue of the Brillouin phonon modes in the crystalline solid. The detailed knowledge of the $Q$-dependent shape and position of these excitations yields - if compared with theoretical models and/or computer simulations - important information about interatomic and intermolecular forces in the liquid, and about the physical processes which lead to the decay of the collective in-phase oscillations. However, the widths of the Brillouin and the central quasielastic line in the scattering law $S(Q, E)$ considerably increase with rising $Q$. Hence, at sufficiently high momentum transfer, all the lines are expected to merge into one broad intensity distribution where the collective modes are no longer discernable. It is hence advisable to perform Brillouin measurements at very small momentum transfers (see e.g. [6]).

While in the $Q \rightarrow 0$ limit the scattering law can be approximated using concepts of classical hydrodynamics, this is no longer possible at momentum transfers where conventional neutron scattering experiments are being carried out. At these $Q$ 's, concepts from extended hydrodynamics, a phenomenologically based extension of classical hydrodynamics, are usually being employed [7]. In this context the question arises, up to which $Q$-value the classical theory is applicable and $S(Q, E)$ is determined by the bulk transport coefficients, and if there is a range which is characterized by the transition between the two regimes. Hence, Neutron Brillouin Scattering (NBS) experiments are the only possibility to answer this question and to test the validity of the corresponding theoretical approaches [8].

Another interesting research area, which can take advantage of Brillouin spectroscopy, is the investigation of magnetic dynamics in condensed phases. Indeed, since the magnetic moments of the atoms are related to their electronic structure, the scattering intensity is determined by the respective electron-magnetic form factor which reaches its maximum at $Q=0$ [9]. Also, the discrimination between ordinary phonon scattering and scattering from magnetic excitations can unambiguously be performed only in the low- $Q$ limit, where the phonon-neutron scattering amplitude vanishes. Hence, it is eligible to carry out inelastic magnetic scattering at as small as possible $Q$-values.

Spectra at low momentum transfer are also highly desirable if the microscopic dynamics close to critical fluctuations is investigated. The maximum intensity of the scattering from critical fluctuations or fluctuations close to second order phase transitions is located at $Q=0$. Usually, transport phenomena diverge or disappear with a characteristic critical exponent when such a transition is approached. In order to investigate the fluid dynamics upon approaching such a transition, it is essential to measure at small $Q$-values, as the accuracy of the measurements increases with decreasing momentum transfer [10]. Again, BRISP is perfectly suited for such investigations.

Small $Q$ values are also essential in the research domain of biological, oligomeric or macromolecular systems. Indeed, proteins and enzymes are known to fulfill their biological functionality only if they are capable of moving. The study of the dynamical behavior of such systems, which occurs 
on a huge range of space- and timescales, from some tenth of nanometer to a few micrometers and from picoseconds to several seconds, is therefore of major interest. In such organized systems made of large molecules, the static structure factor has its main maximum at very small $Q$-values. Hence, a considerable portion of the molecular dynamics takes place at thermal energies and at small momentum transfers. Due to the cooperative and coordinated character of many of such molecular motions, the role of collective coherent fluctuations is much debated and their investigation is highly desirable. In order to reliably measure coherent scattering spectra of such molecular systems, the use of a NBS spectrometer is also indispensable.

There is undoubtedly a still larger number of examples, where the measurement of energy spectra at low $Q$ would be highly welcome. However, the full listing of all these scientific issues would certainly go beyond the scope of this article. In the following, after a brief technical description of the instrument, we will rather focus on the very first experimental spectra obtained by the new NBS spectrometer BRISP for few selected systems. The challenging construction phase was indeed concluded during 2006, and commissioning began with the first test experiments. The spectrometer was finally opened to external users by the end of 2006, when the first call for proposal was sent out.

\section{Instrument outline and working principle}

The Brillouin spectrometer BRISP is the first NBS instrument optimized for the study of disordered and magnetic systems by means of thermal neutrons. While for a detailed and extensive technical description we refer the reader to a more specific paper [5], in what follows we briefly outline the main components and the working principle of the instrument.

BRISP is the so-called hybrid-geometry spectrometer, where a crystal-monochromator fixes the incident neutron energy, and a chopper system defines the neutron final energy by time-offlight analysis. The access to the low- $Q$ inelastic region is made possible by the combination of such instrumental geometry with an optimized two-dimensional small-angle detector. Figure 2

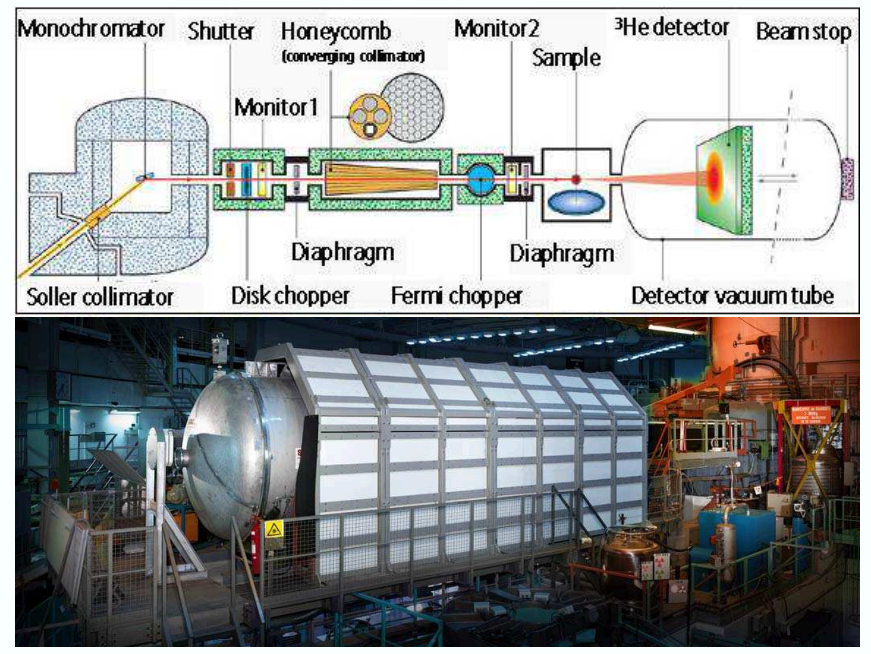

Figure 2. Top: schematic layout of the BRISP beam line. Bottom: a real view of the spectrometer, which sits on a $4 \mathrm{~m}$ high stainless-steel platform in the ILL reactor hall. In the foreground, the big detector shielding is visible. The whole beam line is $18 \mathrm{~m}$ long.

shows a block diagram of the spectrometer with its basic components. The inclined neutron beam emerging from the reactor is first collimated by a standard Soller collimator $\left(0.4^{\circ}\right.$ divergence) before impinging, with a Bragg angle of $17.1^{\circ}$, on the focussing monochromator having an average mosaic spread of $0.4^{\circ}$. Three values of incident energy can be selected by the different monochromator crystals which are currently available. Pyrolytic graphite, exploiting either the (002) or the (004) 
reflections, produces incident neutrons of 20.9 and 83.7 meV respectively, while $\mathrm{Cu}(111)$ is used at the intermediate incident energy of $54.1 \mathrm{meV}$. A rotating disk chopper with eight windows is used to minimize background neutrons from the beam and, by a proper phasing to a Fermi chopper further down-stream, to suppress contaminations by higher-order monochromator reflections. The long neutron pulses produced by the disk chopper travel then through a $2 \mathrm{~m}$ long honeycomb collimator [11], which causes the neutron beam to converge at the detector position. Neutrons leaving the collimator are finally reduced to short pulses by the fast-rotating Fermi chopper. Neutrons scattered by the sample are then collected by the multi-tube position-sensitive ${ }^{3} \mathrm{He}$ detector, covering a $2.1 \mathrm{~m}^{2}$ area around the direct beam. Different small-angle ranges, between 0.6 and $15^{\circ}$, can be continuously covered by varying the sample-to-detector distance between 1.5 and $6 \mathrm{~m}$. The above technical characteristics determine the final performance of the spectrometer in terms of both accessible dynamical range and energy-wavevector resolution. The portion of dynamical range covered by BRISP is reported in figure 3 and compared with the $Q-E$ regions accessible by other experimental techniques. Considering the various possible instrumental configurations, on the whole the spectrometer can span energy exchanges from 0.6 to about $100 \mathrm{meV}$ and elastic momentum transfers from 0.04 to $8 \AA^{-1}$ with resolutions of $0.02 \leqslant \Delta Q \leqslant 0.05 \AA^{-1}$ and $3 \% \leqslant \Delta E / E_{0} \leqslant 4 \%$ where $E_{0}$ is the neutron incident energy. This makes possible the collection of inelastic spectra in the thermal region at $Q$-values ranging from about 0.2 to $1.8 \AA^{-1}$.

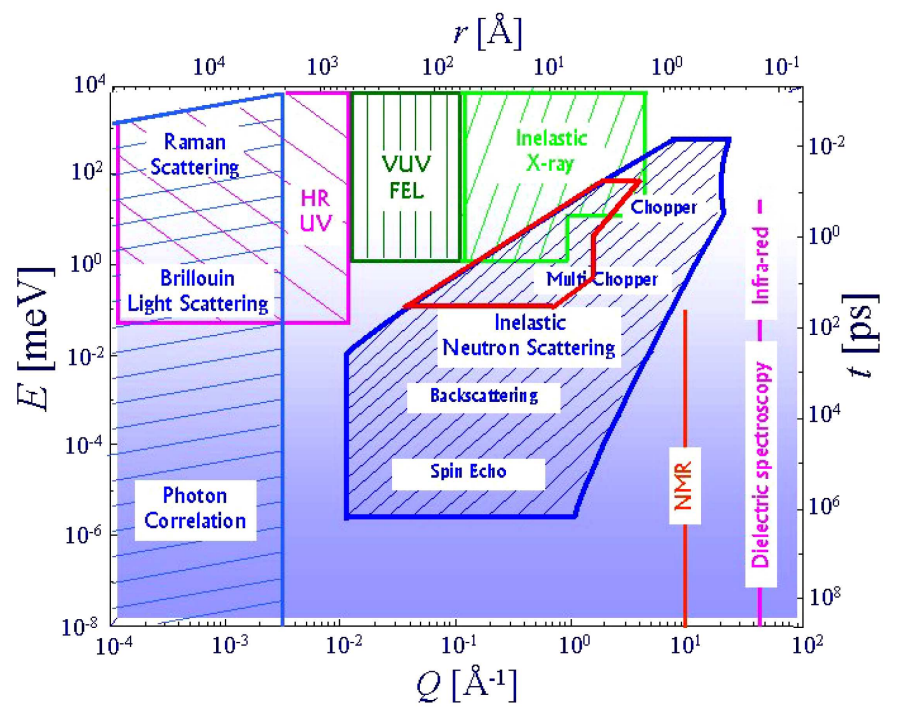

Figure 3. Dynamical ranges accessible by the many spectroscopic techniques nowadays available. The region defined by the red line shows the dynamical range BRISP is optimized for.

As suggested by figure 3, other thermal neutron instruments can cover the dynamical region of BRISP with comparable resolutions, namely three-axis spectrometers (TAS). These instruments provide an extremely flexible choice of incident and final neutron energies, scattering angles and collimations, thus spanning a much wider kinematic region and eventually providing optimal configurations for NBS studies. Nevertheless, for intrinsic technical reasons, TAS suffer from very long acquisition times, which make this method particularly inefficient for the study of isotropic samples, especially when many $Q$ values at several thermodynamic states are to be investigated. In fact, TAS are extremely powerful in performing what can be thought of as a surgical cut of the dynamical plane, as it happens, for instance, when determining the dispersion curves of crystal phonons, which are well localized along a defined path in the $Q-E$ plane. On the contrary, thermal Brillouin modes in disordered systems are usually broad and produce an intrinsically low scattering intensity. In such cases, the time-of-flight technique is more efficient, since it provides contemporaneous access to the whole available dynamical range. 


\section{Some exemplary experiments}

To illustrate the potentialities of BRISP, herein below we present some spectra of the very first samples measured by users during the last year.

A very good benchmark experiment is the measurement of the low- $Q$ dynamic structure factor of liquid lead at $630 \mathrm{~K}$. Indeed $\mathrm{Pb}$ is a particularly favorable system due to its quite large and almost completely coherent cross-section $\left(\sigma_{\mathrm{sc}}^{\mathrm{coh}}=11.115\right.$ barns, $\sigma_{\mathrm{sc}}^{\text {inc }}=0.003$ barns $)$, along with its very small absorption cross-section ( $\sigma_{\mathrm{abs}}=0.1$ barns for $83.7 \mathrm{meV}$ incident neutrons). Such scattering properties permit the use of massive samples, which have the advantage of increasing the scattered intensity without necessarily trading off a poor transmission, provided the sample geometry is thoughtfully tailored. On the other hand, due to the large atomic number of $\operatorname{Pb}(Z=82)$, such an experiment performed using IXS would be severely limited by strong absorption. A final advantage arises from the speed of sound in liquid lead, which amounts to only $1770 \mathrm{~m} / \mathrm{s}$, and therefore the kinematical constraints on the accessible dynamical range with neutron spectroscopy are easily satisfied. Therefore several neutron experiments on liquid lead have already been carried out, one of which aimed at determining the Brillouin modes in the thermal region at $Q$-values between 0.4 and $1.2 \AA^{-1}$ [12]. Therefore a new measurement on this system is a good way of testing the performances of BRISP against the existing results.

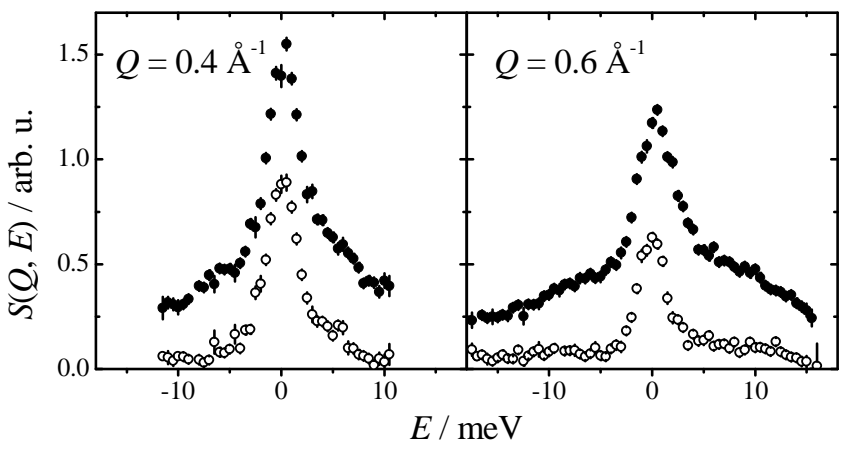

Figure 4. Inelastic spectrum of liquid lead measured on BRISP at 0.4 and $0.6 \AA^{-1}$ (full circles). The spectrum of the empty cell is also plotted for comparison (empty circles).

In the experiment herewith presented, a sample of $136 \mathrm{~g}$ was placed in a slab-shaped $\mathrm{Al}$ cell, having a cross-section of $4 \mathrm{~cm} \times 5 \mathrm{~cm}$ and a thickness of $0.6 \mathrm{~cm}$ along the beam direction. At the employed wavelength of $0.9885 \AA(83.7 \mathrm{meV})$, this yields a transmission value of 0.80 and less than $5 \%$ multiple scattering contribution to the total (integrated over all the energies and wavevectors) cross-section. At the chosen incident wavelength, the energy resolution amounts to $2.7 \mathrm{meV}$ at the elastic peak. In 6 days of acquisition time, the spectra of both the sample and the empty cell were collected at 16 different $Q$-values, between 0.1 and $1.6 \AA^{-1}$. As an example, the spectra normalized to the same counting time are presented in figure 4 at two intermediate values of the exchanged wavevector, namely 0.4 and $0.6 \AA^{-1}$. Apparently, from comparing the empty cell with the sample spectra, a clear inelastic signal is present and is well measurable in liquid lead. Regardless of their physical interpretation, which is beyond the scope of this paper, the presented data indicate that BRISP is well capable of measuring the low- $Q$ dynamic structure factor of a liquid metal in the thermal region, and that this is feasible in definitely competitive acquisition times.

As a second example of application of BRISP to the study of amorphous systems, we turn to the glassy material $\mathrm{GeSe}_{2}$. Acoustic high-frequency modes in glasses are being widely studied in the attempt to understand their connection with the well-known dynamical and thermodynamical anomalies of these systems, lately with particular interest to possible dynamical differences between fragile and strong glasses. Within this framework, a systematic study of the strong glass-former $\mathrm{GeSe}_{2}$ has been recently undertaken. The first attempt to measure its low- $Q$ dynamic structure factor took place on BRISP in May 2007 [13]. 
The speed of sound in vitreous $\mathrm{GeSe}_{2}$ ranges from 1700 to $2200 \mathrm{~m} / \mathrm{s}$, which falls well within the dynamical range of BRISP. Nevertheless, at variance with the case of liquid lead, this is a more disadvantaged case due to the less favorable ratio between scattering and absorption cross-sections: the relevant values, referred to one $\mathrm{GeSe}_{2}$ molecule, are $\sigma_{\mathrm{sc}}^{\mathrm{coh}}=24.38$ barns, $\sigma_{\mathrm{sc}}^{\text {inc }}=0.82$ barns, and $\sigma_{\mathrm{abs}}=14$ barns for $83.7 \mathrm{meV}$ incident neutrons. In addition, the lengthy production process of amorphous $\mathrm{GeSe}_{2}$ remarkably limits the quantity of available sample. Finally, about $45 \mathrm{~g}$ of sample were measured in a slab-shaped $\mathrm{Al}$ cell of $5 \mathrm{~cm}$ width, $3 \mathrm{~cm}$ height and $1 \mathrm{~cm}$ thickness along the beam, which yields a transmission of 0.74 at the chosen wavelength of $0.9885 \AA$. The acquisition of the inelastic spectra at 16 different $Q$-values, between 0.1 and $1.6 \AA^{-1}$, lasted 60 hours for $\mathrm{GeSe}_{2}$ and 27 hours for its empty cell. The spectra at 0.5 and $0.6 \AA^{-1}$, corrected for empty cell contributions, are shown in figure 5. Despite the less favorable scattering properties of $\mathrm{GeSe}_{2}$, a well defined inelastic signal emerges from the background.

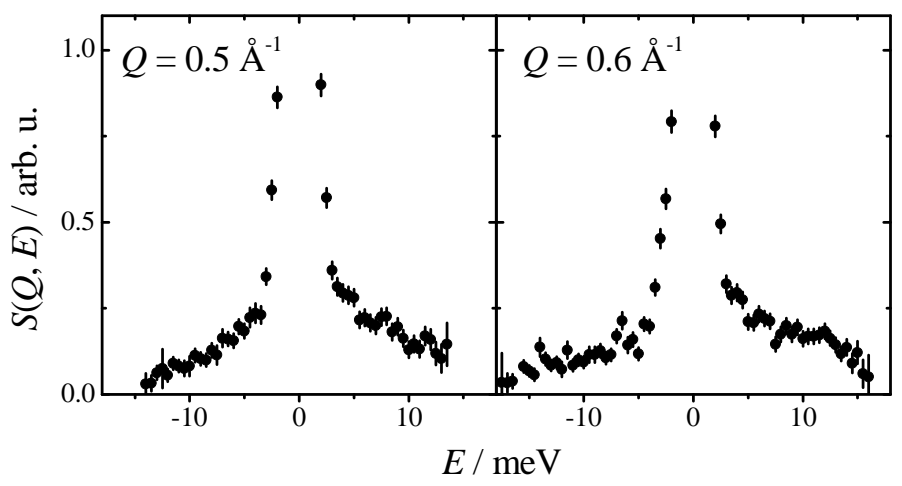

Figure 5. Inelastic spectrum of vitreous $\mathrm{GeSe}_{2}$ measured at 0.5 and $0.6 \AA^{-1}$. The data are corrected for empty cell contributions.

The last example we wish to present, which is the most exotic and experimentally the most difficult one, concerns a biological system. Neutrons are indeed a particularly well-suited probe for delicate organic molecules, as they cause much less radiation damages than x-rays. The biological functionality of proteins is known to be determined by their dynamical properties, which in turn are triggered by and strongly coupled to those of the protein hydration shell, in the absence of which no protein can work. The experiment presented in the following was therefore aimed at studying the dynamical behaviour of the hydration shell of the widely-studied protein Ribonuclease (RNase). While a previous attempt of measuring the collective modes in a protein powder exists [14], this is the first pioneering experiment aimed at observing possible coherent excitations in the sole hydration shell.

In order to single out the contribution of hydration water alone, two samples had to be measured: a first one made of a completely dry RNase powder, and a second one obtained by hydrating the previous dry powder with deuterated water, at the hydration level of $h=1 \mathrm{~g}$ of water per $\mathrm{g}$ of protein. The choice of deuterated water, instead of $\mathrm{H}_{2} \mathrm{O}$, is imposed by the huge incoherent crosssection of hydrogen $\left(\sigma_{\mathrm{sc}}^{\mathrm{coh}}=1.76\right.$ barns, $\sigma_{\mathrm{sc}}^{\text {inc }}=80.26$ barns $)$ which would prevent us from observing any possible coherent collective excitation in the hydration shell. On the contrary, deuterium atoms have a predominantly coherent scattering cross-section $\left(\sigma_{\mathrm{sc}}^{\mathrm{coh}}=5.59\right.$ barns, $\sigma_{\mathrm{sc}}^{\text {inc }}=2.05$ barns $)$, and thus heavy water results to be much more suited for this kind of experiments. With this atomic composition of the sample, the coherent inelastic spectrum of the hydration shell can be obtained by properly subtracting the spectrum of dry RNase to that of wet RNase. Apart from carefully choosing the corrected subtraction procedure, the main experimental difficulty arises from the fact that the RNase protein itself is largely made of hydrogen atoms. Luckily enough, the instrumental configuration can be properly chosen, such that the resulting strong incoherent signal remains confined within the elastic part of the spectrum, and does not spoil the inelastic region of interest. Considering the high-frequency speed of sound in bulk water (3200 m/s) [15], the optimal BRISP 
configuration turned out to be the one employing $83.7 \mathrm{meV}$ incident neutrons with an elastic energy resolution of $2.7 \mathrm{meV}$. Besides its incoherent nature, the large absolute value of the hydrogen crosssection is also responsible for a fast transmission drop with increasing sample thickness and for a remarkable multiple scattering contribution. Both effects must be carefully considered when choosing the sample geometry and when performing the data reduction.

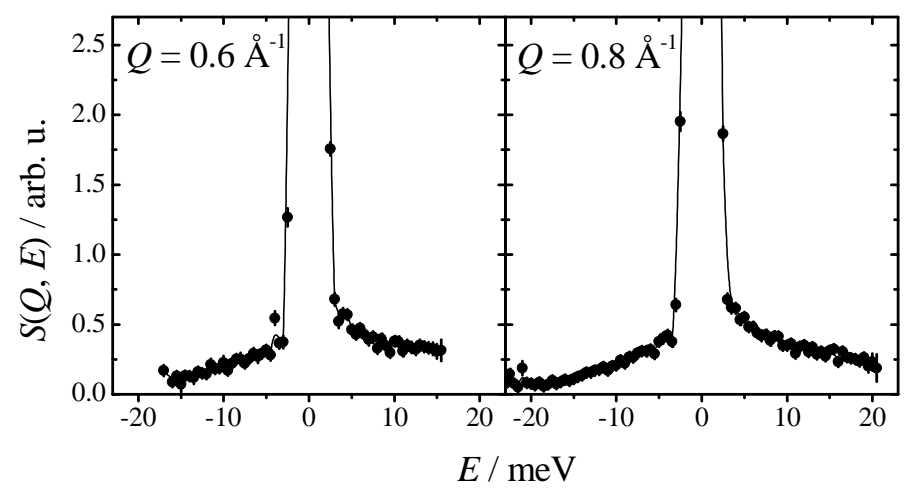

Figure 6. Dynamic structure factor of RNase hydration water, measured on BRISP at 0.6 and $0.8 \AA^{-1}$. The full line is a guide to the eye.

Without entering further technical details, both the dry and the wet sample were measured for 4 days in a slab-shaped $\mathrm{Al}$ cell of dimensions $5 \mathrm{~cm} \times 3 \mathrm{~cm} \times 0.3 \mathrm{~cm}$. The acquired data were carefully corrected for background and cell contribution, detector efficiency, multiple scattering, sample selfabsorption. The so-obtained dynamic structure factor of the dry sample was then subtracted from that of the wet one. The resulting spectrum is plotted in figure 6 for two values of the exchanged wavevector, namely 0.6 and $0.8 \AA^{-1}$ [16]. As it can be observed, a neat positive inelastic signal is visible after subtraction of all the undesired contributions and can be safely ascribed to the dynamical behaviour of the sole hydration water. Such a result represents one example among a number of pioneering experiments on a wider class of complex molecular systems, which were considered of difficult feasibility until now, but might be successfully carried out in the future.

\section{Conclusions}

The presented experimental results clearly demonstrate the functional capabilities of the new Brillouin Spectrometer BRISP. The accessibility of inelastic spectra in the lower $Q$-range and at thermal exchanged energies is unique among other neutron time-of-flight spectrometers. In terms of energy resolution and accessible $Q-E$ region, BRISP is definitely competitive with both IXS and TAS. With respect to the latter, it represents an efficient method in cases where a quick and wide survey of the whole low- $Q$ dynamic structure factor is needed. Moreover, by exploiting the peculiar isotope dependence of neutron cross-sections, BRISP can provide a unique possibility of extracting complementary information with respect to x-rays.

In fact, the experiments carried out so far have proven that possible scientific applications range from the investigation of simple and complex molecular liquids, via macromolecular and biological systems, towards systems with dynamical magnetic properties. Moreover, the present instrument performances are expected to be further improved by the foreseen future upgrades.

We are convinced, that this new-type neutron spectrometer is an appreciated enrichment of the available scientific methods in the field of microscopic particle dynamics. We look forward to a large number of new and appealing experiments and we expect that BRISP will positively contribute to the attempt to answer new and longstanding scientific questions. 


\section{Acknowledgements}

The Institut Laue-Langevin is gratefully acknowledged for providing both neutrons and prompt technical assistance. We thank all the members of the BRISP CRG for their contributions to the development of BRISP. In particular, we wish to thank F. Barocchi for promoting the project in Italy, T. Gahl for the construction of the large bidimensional detector, F. Formisano and L. Sani for the development of the data formatting software, A. Cunsolo for his help during the beamline installation, A. De Francesco and A. Laloni for their reliable management of the instrument since the first months of operation, and E. Guarini for her technical support in Italy. A warm and grateful acknowledgment is finally addressed to the technicians of the Physics Department of the University of Perugia (E. Babucci, D. Aisa, S. Aisa and A. Piluso) for their invaluable support to the spectrometer construction, and to the technicians of the University of Marburg (W. Kroeschel and M. Freundorfer) for their efforts concerning the completion of the position sensitive detector.

\section{References}

1. Sinn H., Sette F., Bergmann U., Halcousis Ch., Krisch M., Verbeni R., Burkel E., Phys. Rev. Lett., 1997, 78, 12.

2. Pilgrim W.-C., Hosokawa S., Saggau H., Sinn H., Burkel E., J. Noncryst. Solids, 1999, 250-252, 96.

3. Copley J.R.D., Rowe J.M., Phys. Rev. Lett., 1974, 32, 49.

4. Pilgrim W.-C., Morkel C., J.Phys.: Condens. Matter, 2006, 18, R585.

5. Aisa D., et al., Nucl. Instr. and Meth., 2005, 544, 620.

6. Suck J.-B., Int. J. Mod. Phys. B, 1993, 7, 3003.

7. Boon J.P., Yip S. Molecular Hydrodynamics. New-York, Dover, 1974.

8. Bafile U., et al., Phys. Rev. Lett., 1990, 65, 2394.

9. Lovesey S. Theory of Neutron Scattering from Condensed Matter. Oxford University Press, 1984.

10. Chahid A., et al. Studsvik Ann. Rep., 1996.

11. Petrillo C., Guarini E., Formisano F., Sacchetti F., Babucci E., Campeggi C., Nucl. Instr. and Meth. A, 2002, 489, 304.

12. Söderström O., Copley J.R.D., Suck J.-B., Dorner B., J. Phys. F: Metal Phys., 1980, 10, L151 and references therein.

13. Orsingher L., Violini N., Baldi G., Fontana A. Private communication, 2007.

14. Bellissant-Funel M.-C., Teixeira J., Chen S.H., Dorner B., Middendorf H.D., Crespi H.L., Biophys. J., 1989, 56, 713 .

15. Sacchetti F., Suck J.-B., Petrillo C., Dorner B., Phys. Rev. E, 2004, 69, 0612031 and references therein.

16. Orecchini A., Paciaroni A., Petrillo C., Sacchetti F., submitted. 


\title{
BRISP, новий малокутовий нейтронний спектрометр для дослідження колективної динаміки у невпорядкованій речовині
}

\author{
А.Ореккіні ${ }^{1}$, В.-Х.Пільґрім², К.Петрілло ${ }^{1}$, Й.-Б.Сук ${ }^{3}$, Ф.Саккетті ${ }^{1}$ \\ 1 Фізичний факультет, Університет Перуджі, Перуджя, Італія \\ 2 Фізична хімія, Філіпс університет Марбурґа, Марбурґ, Німеччина \\ 3 Інститут фізики, Технічний університет Хемнітца, Хемнітц, Німеччина
}

Отримано 20 листопада 2007 р.

Подано короткий опис нового нейтронного брілюенівського спектрометра BRISP, що інсталювався протягом останніх років та недавно почав діяти в Інституті Лауе-Ланжевена у Ґреноблі (Франція). Описано основні технічні особливості та представлено деякі перші експериментальні результати, що демонструють наявні можливості цього малокутового непружнього спектрометра нового типу. В майбутньому передбачаються та очікуються вдосконалення для майбутнього покращення роботи інструмента.

Ключові слова: інструменти нейтронного розсіяння, час польоту, малокутові спектрометри

PACS: 07.05. Fb, 29.30. Hs 